\title{
The study of deceased organ donation during the COVID-19 pandemic in Korea
}

\author{
Jaesook Oh, Jina Park, Jeongrim Lee, Insung Moon
}

Korea Organ Donation Agency, Seoul, Korea

Background: In 2020, the COVID-19 pandemic caused the global health-care system to collapse and continuously affect organ donation and transplantation. Organ donation and transplantation activity in all countries showed an overall decrease at the beginning of the pandemic due to increased health-care service utilization, this decrease stabilized as establishing response system after the first pandemic. Unlike this trend, in South Korea, organ donors have increased to 288 (January to July 2020) and showed a reduction trend from July.

Methods: In this study, we examined the effect of the COVID-19 pandemic on deceased organ donation by analyzing the number of referred potential organ donors.

Results: The first cases of COVID-19 in Korea were reported at the mid-January 2020 and gradually increased by March 2020. In this period, the outbreak in Daegu and Gyeongsangbuk-do province accounted for more than $90 \%$ of all COVID-19 cases. From early April to mid-August, the number of COVID-19 cases were decreased, and the government relaxed the quarantine rule. We noted that in the first half of 2020, the number of brain-dead patients referred to Korea Organ Donation Agency (KODA) decreased by $16 \%$ compared with the previous year; the family approach and consent rate increased to $83.8 \%$ and $37.4 \%$, respectively, $4.4 \%$ and $5.4 \%$ compared with the previous year, respectively. There are some reasons for this trend. First, the healthcare system in the greater Seoul area worked normally because COVID-19 infection occurred only in few areas like Daegu. The potential braindead patients stayed longer in hospitals due to hospital transfer constraints, it extended in-hospital patient time and increased the opportunities to interview the patient's family about organ donation by medical staff or organ procurement coordinator. And two series of the drama about organ donation also affected increasing the family approach and consent rate. However, the case of COVID-19 turned to increase even in the greater Seoul area after the massive rally on August 15 . The new daily cases reached 5,800 , and the government announced tougher social distancing. The Korea had highest number of infections with metropolitan area in October and November, but the government eased social distancing.

Conclusions: In the second half of 2020, the number of brain-dead patients referred to KODA decreased to 933 cases which 72 cases fell compared with the previous year; the number of organ donors slightly reduced to 190 cases which seven cases dropped with the year earlier. Although the rate of patient's family approach slightly increased $(2019,80.7 \% \rightarrow 2020,83 \%)$ in referred potential brain-dead patients, the organ donation rate was decreased due to the decline of consent rate. In the first half of 2020, despite COVID-19 hurdles, deceased organ donation increased by building an effort of the government-society-KODA and hospitals, but in the other half of 2020 , the organ donation rate turned to decreased. In 2021, the government announced an organ donation initiative; it's time to join the medical staff, hospitals, and society to promote and implement the initiative.

Corresponding author: Jaesook Oh

E-mail: js.oh@koda1458.kr

(C) The Korean Society for Transplantation

This is an Open Access article distributed under the terms of the Creative Commons Attribution Non-Commercial License (http://creativecommons.org/licenses/by-nc/4.0/) which permits unrestricted non-commercial use, distribution, and reproduction in any medium, provided the original work is properly cited. 